\title{
EXPERIMENT TO MEASURE HARD SOLAR AND CELESTIAL X-RAYS FROM THE FIFTH ORBITING SOLAR OBSERVATORY
}

\author{
K. J. FROST, B. R. DENNIS and R. J. LENCHO \\ Laboratory for Solar Physics, NASA-Goddard Space Flight Center, Greenbelt, U.S.A.
}

\begin{abstract}
In this paper we describe an experiment designed to measure solar and celestial X-rays in the energy range between 15 to $250 \mathrm{keV}$. The experiment was flown on the fifth Orbiting Solar Observatory which was launched on 22 January 1969 . Up to the time of this writing the instrument continues to operate satisfactorily.
\end{abstract}

\section{Scientific Objectives}

The objectives for measuring solar X-rays are:

(1) Measure the spectrum of Solar X-ray bursts as a function of time throughout the duration of the bursts.

(2) Conduct a search for a weak, constantly present and/or activity-dependent component of solar $\mathrm{X}$ rays in the 15 to $250 \mathrm{keV}$ range for successive solar rotations during the lifetime of the spacecraft.

The objectives in measuring celestial X-rays are:

(1) Measure the intensity, spectrum and spatial distribution of diffuse celestial X-rays.

(2) Measure the intensity, spectrum and possible temporal variation of those point sources that can be isolated with the rather broad field of view $\left(40^{\circ} \mathrm{FWHM}\right)$ of the detector. The Crab Nebula is the only source for which this is easily done.

\section{Detector}

A schematic of the detector is presented in Figure 1. The central element of the detector is a disc of CsI (Na) of $0.64 \mathrm{~cm}$ thickness and $71 \mathrm{~cm}^{2}$ area. This crystal is viewed by two photomultiplier tubes which are operated in coincidence to achieve a noise-free threshold at $15 \mathrm{keV}$. The central crystal assembly is enclosed in an anticoincidence shield of $\mathrm{CsI}(\mathrm{Na})$ which provides the desired collimation of $39^{\circ} \mathrm{FWHM}$. The geometric factor of the instrument is $34 \mathrm{~cm}^{2}$ ster. The low background characteristics of the high- $Z$, actively shielded detector permits the scientific objectives of the experiment to be achieved with a high level of sensitivity. The thickness of the shield is adequate to insure that at $250 \mathrm{keV} 70 \%$ of the flux measured in the central crystal enters through the aperture with the remainder leaking through the anticoincidence shield.

The efficiency of the detector is presented in Figure 2. The rapid decrease in efficiency below $30 \mathrm{keV}$ is caused by absorption in the two aluminum windows of 0.15 $\mathrm{g} \mathrm{cm}^{-2}$ total thickness (Figure 1). This was intentionally designed into the experiment to suppress pulse pile-up distortion of flare spectra by an intense flux of soft photons. 


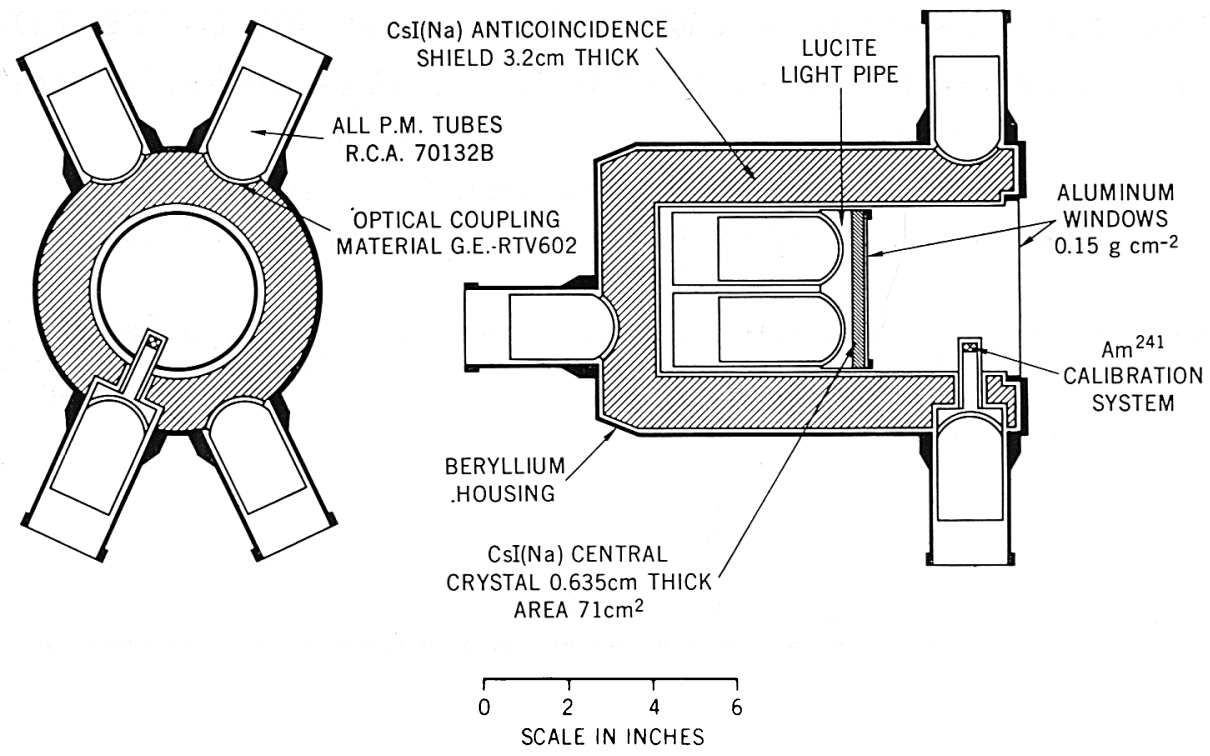

Fig. 1. OSO-5 15-250 keV X-Ray detector.

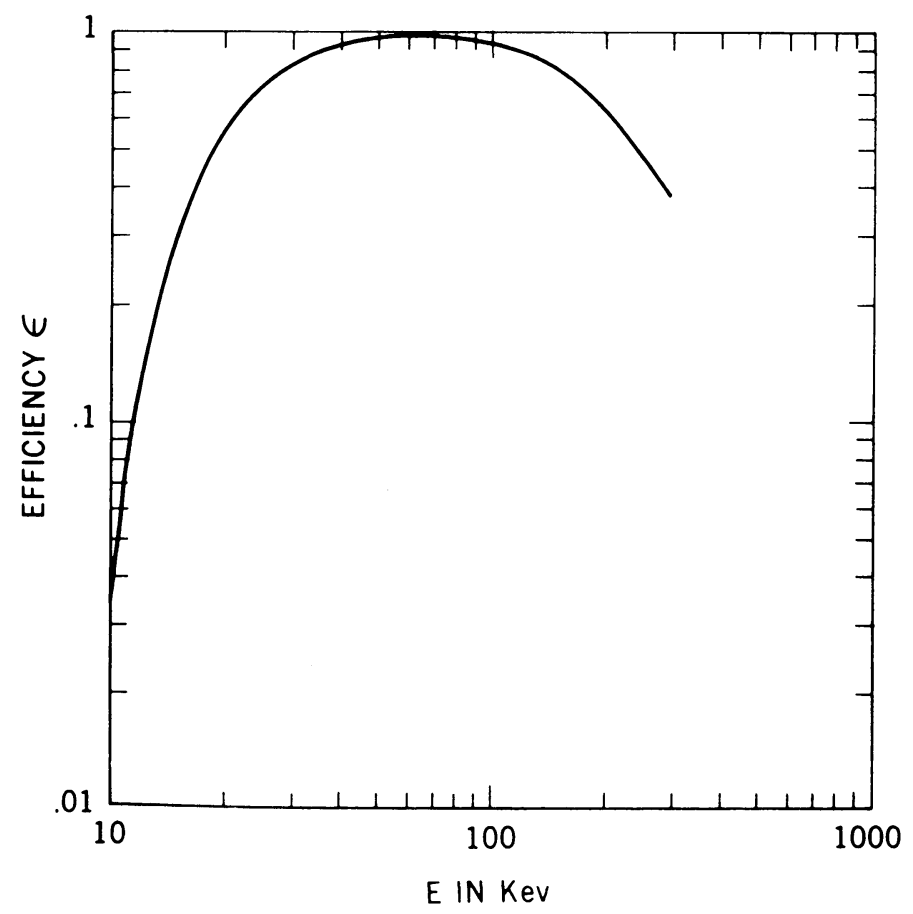

Fig. 2. Efficiency of detector excluding effects of double coincidence and escape corrections. 
A further decrease in efficiency below $30 \mathrm{keV}$ occurs because of the coincidence requirement on the central crystal phototubes - not every photon below $30 \mathrm{keV}$ produces a signal in both tubes above threshold. This effect is evaluated in orbit by turning the coincidence requirement on and off by command and noting the difference in the counting rate at low energy.

Long term, verifiable stability is an indispensable requirement for fulfilling the scientific objectives described earlier. Calibration of the instrument is accomplished through the use of the isotope $\mathrm{Am}^{241}$ which emits a $60 \mathrm{keV}$ photon in coincidence with an alpha particle of about $5 \mathrm{MeV}$ energy. The $\mathrm{Am}^{241}$ is dissolved in a piece of plastic scintillator which is mounted on a light pipe and positioned as indicated in Figure 1. The alpha particles lose all their energy in the plastic scintillator and in so doing produce a large light burst which is detected through the light pipe by a photomultiplier tube. The $60 \mathrm{keV}$ photons escape with reasonable efficiency from the scintillator in all directions. The alpha particle pulse from the phototubes can be used as a tag on the $60 \mathrm{keV}$ photons. By operating the central crystal tube in coincidence with the tube viewing the calibration source, a spectrum composed exclusively of $60 \mathrm{keV}$ photons is obtained. By operating in anticoincidence with the calibration photoube the data collected will have no contribution from the calibration photons. If the position of the calibration photopeak shifts, the gain of the central crystal linear amplifier can be increased or decreased by ground command to compensate for the drift. Eight levels of gain change are available permitting three decrements and five increments of gain. Figure 3 presents two calibration spectra taken approximately 17 months apart. Each spectrum is plotted with only one-fourth of the calibration data available per day. Only one increment of gain was required in October 1969 to maintain the central crystal stability indicated in Figure 3.

The threshold of the anticoincidence shield was set between 100 and $150 \mathrm{keV}$ at launch. The stability of this threshold is verified in orbit by monitoring the instrumental dead time which is primarily a function of the count rate in the anticoincidence shield. In orbit the dead time varies as a function latitude and longitude in an approximately cyclic and predictable way from day-to-day. A change in the anticoincidence threshold would shift the daily pattern of dead time to generally higher or lower values. The linear amplifier for the shield can be changed in gain by command to compensate for drifts in threshold.

\section{Observational Format}

There are three modes of operation for this experiment, two day modes and one night mode. The day modes can be understood by referring to Figure 4 which shows the position of the experiment in the rotating section of the spacecraft. As the spacecraft rotates in a counter-clockwise direction as viewed in Figure 4, the scan of the strip of sky in the plane of the wheel is divided into 9 sectors of $40 \mathrm{deg}$ each. This division is accomplished by 9 photo-diodes, 3 in each of the 3 eye-blocks depicted in Figure 4 , positioned $40^{\circ}$ apart and triggering on the Sun. Of course one of these sectors con- 


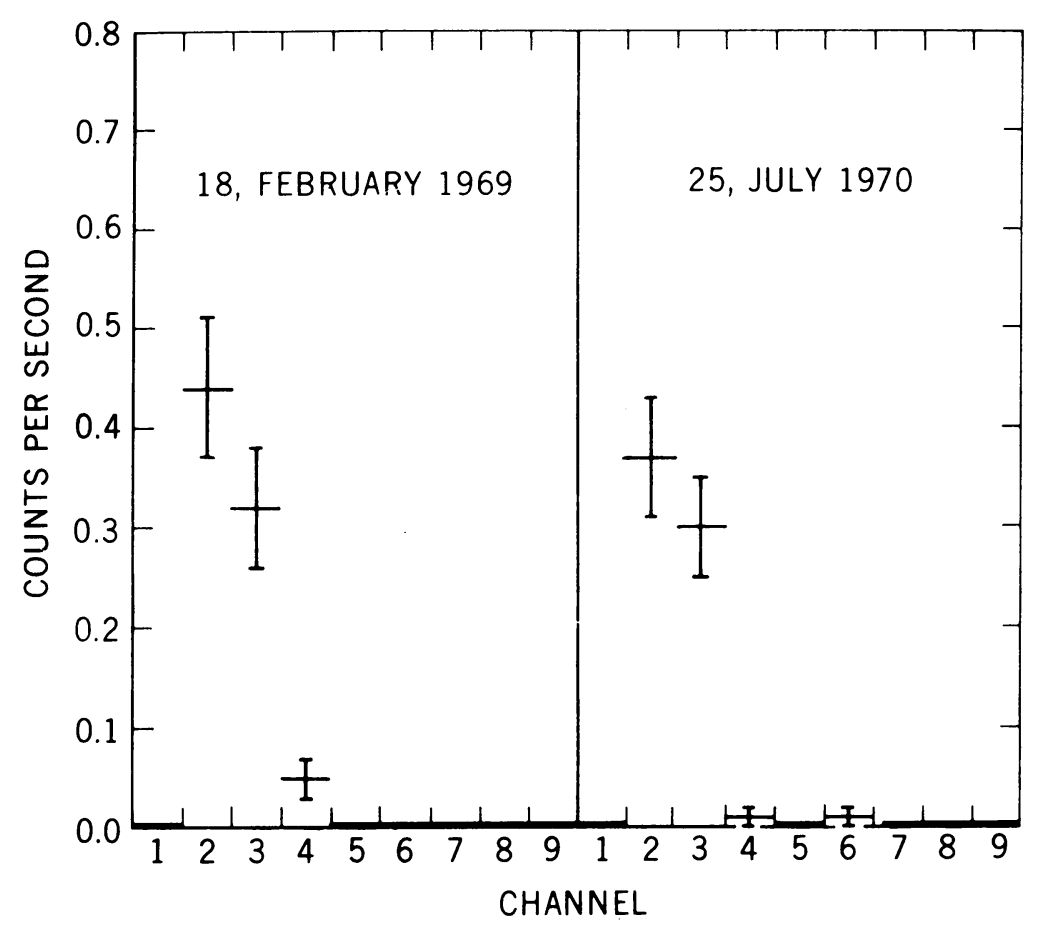

Fig. 3. OSO-5 calibration data $\mathrm{Am}^{2+1}$.

tains the Sun and operation in this sector is referred to as the solar mode. Observations in the remaining 8 sectors are referred to as the day sky scan.

The period of rotation of the spacecraft is nominally $1.8 \mathrm{~s}$. In each rotation data is collected in the solar sector for $0.19 \mathrm{~s}$ with a projected detector area of $54 \mathrm{~cm}^{2}$. The following quantities are measured:

I. PULSE HEIGHT DisTRIBUTION

The 15 to $250 \mathrm{keV}$ interval of the central crystal is subdivided into 9 channels with the energy limits as given in Table I. The scaler capacity of each channel is also listed.

TABLE I

Channel No. Energy in keV Scaler capacity

$\begin{array}{rrr}1 & 14-28 & 4095 \\ 2 & 28-55 & 511 \\ 3 & 55-82 & 127 \\ 4 & 82-111 & 63 \\ 5 & 111-141 & 31 \\ 6 & 141-168 & 31 \\ 7 & 168-200 & 15 \\ 8 & 200-225 & 15 \\ 9 & 225-254 & 15\end{array}$




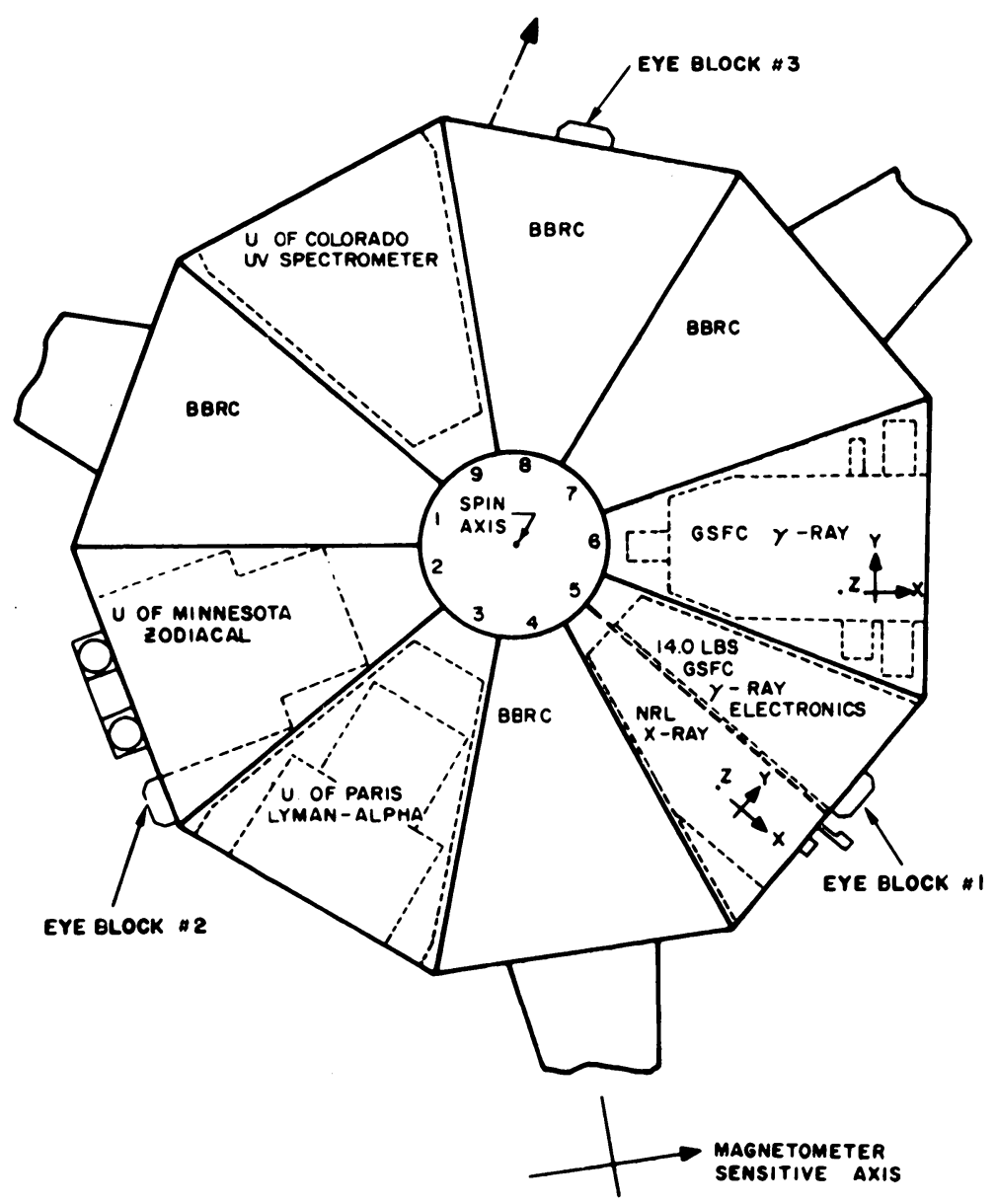

Fig. 4. OSO-5 wheel experiment compartment configuration.

\section{ELAPSED TIME}

The photo-diode pulses signaling the beginning and ending of the solar sector also start and stop respectively the counting by a scaler of pulses from the 800 cycle telemetry clock. The duration of the solar observation or the elapsed time is simply the number of clock pulses counted divided by 800 . The average value of the elapsed time is 0.17 to $0.19 \mathrm{~s}$. There is an exception to this mode of operation. If a solar flare occurs of intensity sufficient to exceed the scaler capacity of any channel before the end of the solar measurement then the counting of clock pulses and the accumulation of all data is stopped by whichever scaler reaches capacity rather than by the later occurring diode pulse. The exact number of counts collected, and the time over which they were collected is consequently preserved and the measurement is not lost due to scaler overflow. 


\section{TOTAL DEAD TIME}

The total instrumental dead time is measured for the duration of each solar observation. The difference between the elapsed time and the dead time is the true observing time.

Observations are made in each of the 8 sky scan sectors by making the same measurements as in the solar sector except that a longer integration time and larger scaler capacities are used and the total counting rates in the shield and central crystal are monitored. The sequence of collecting data begins in the first $40^{\circ}$ sector after the solar sector in the counter-clockwise direction. Data is integrated in this sector over six consecutive rotations. After the sixth rotation the acquired data is shifted to a buffer memory preparatory to being transferred to the spacecraft tape recorder. After the shift, collection of data for six rotations begins in the second $40^{\circ}$ sector after the Sun. During collection of data in this second sector data from the first sector is transferred from the buffer memory to the spacecraft tape recorder. This sequence continues through sectors $3,4,5$ and so on to sector 8 and then repeats continuously throughout
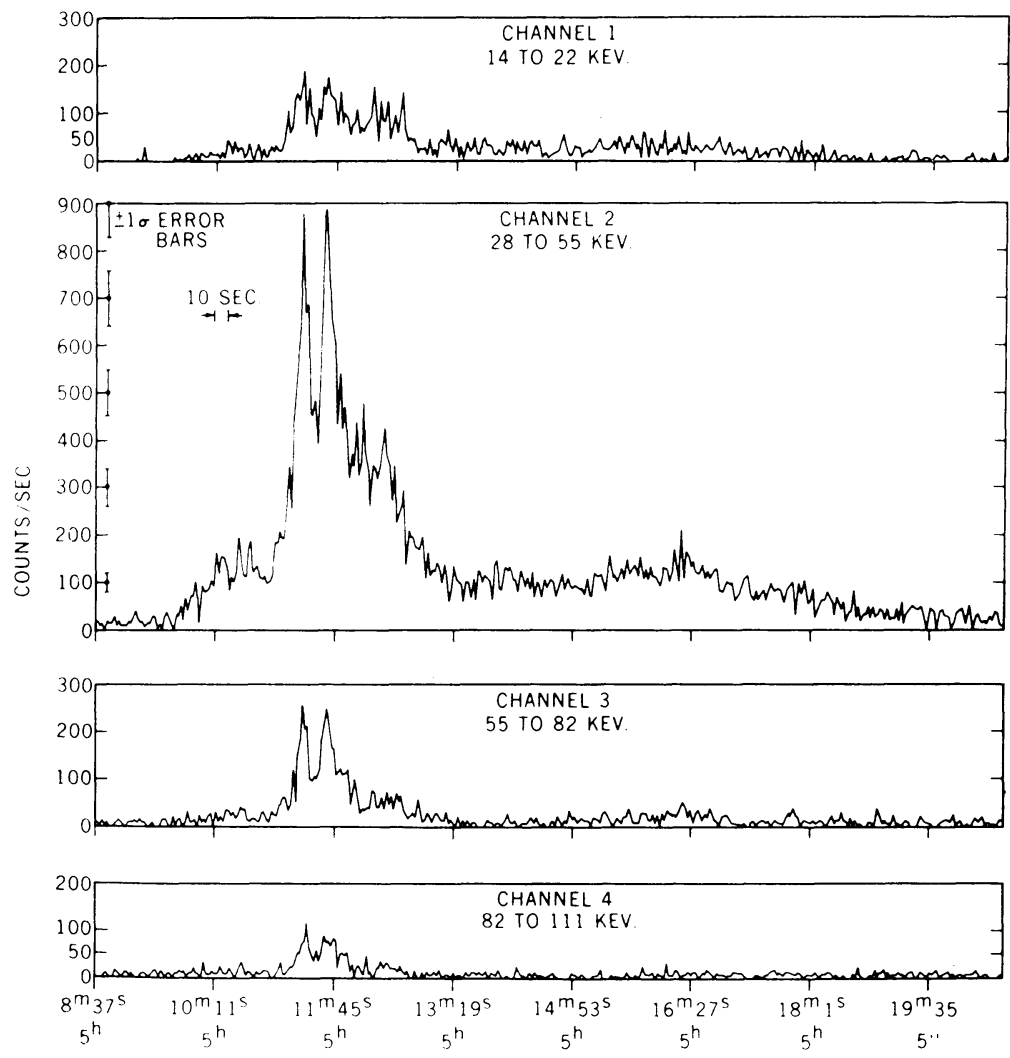

Fig. 5. OSO-5 GSFC wheel exp. X-ray burst, class 2-N flare 2-Feb. $\mathrm{H}_{\mathrm{x}}$ max. $5^{\mathrm{h}} 12^{\mathrm{m}}$ UT. Orbit 159. 
the day portion of the orbit. In each cycle of the sequence, calibration data is collected for six rotations between the rotations of sectors 6 and 7. One cycle of the sequence constitutes a strip scan of the sky and requires $108 \mathrm{~s}$ to produce $10.8 \mathrm{~s}$ of data composed of $1.2 \mathrm{~s}$ for each of the 8 sectors and $1.2 \mathrm{~s}$ of calibration data.

Strip scans of the sky are also made throughout the night phase of the orbit.

The sequencing of data at night is controlled by the rising and falling edges of two telemetry words such that the detector collects $0.28 \mathrm{~s}$ of data every $1.28 \mathrm{~s}$ from whatever region of the sky happens to be in the field of view. The measurements made in each $0.28 \mathrm{~s}$ sample are the same as those made in the solar sector. The attitude of the spinning spacecraft as a function of time is determinable for orbit night and permits us to determine from which region of the sky each observation was collected.

\section{Typical Observation}

In Figure 5 a complex solar X-ray burst is presented which can be used to illustrate the effect of the experiment characteristics on the observations. The low count rate in channel 1, relative to channels 2 and 3, reflects the decrease in efficiency indicated in Figure 3 below $25 \mathrm{keV}$ plus the inefficiency due to the coincidence operation of the central crystal phototubes. In channel 2 a pre-burst and post-burst increase, having gradual rise and fall characteristics, are detected. The low background and large area of the OSO 5 detector permits us to improve the determination of the spectral characteristics of these weaker components. The $2 \mathrm{~s}$ time resolution available in this experiment permits the detection of fast time structure such as the two peaks in Figure 5. Discussion of another OSO-5 X-ray burst similar to that shown in Figure 5 is contained elsewhere in the literature (Frost, 1969).

\section{Reference}

Frost, K. J.: 1969. Astrophys. J. Letters 158, L159. 